BMJ Open Ophthalmology

\title{
Omega-3 fatty acids supplementation protects the retina from age-associated degeneration in aged $\mathrm{C} 57 \mathrm{BL} / 6 \mathrm{~J}$ mice
}

\author{
Ekatherine Prokopiou (D) , ${ }^{1,2}$ Panagiotis Kolovos, ${ }^{1}$ Christos Georgiou, ${ }^{1}$ \\ Maria Kalogerou, ${ }_{1}^{1}$ Louiza Potamiti, ${ }^{3}$ Kleitos Sokratous, ${ }^{3,4}$ Kyriacos Kyriacou, ${ }^{3,5}$ \\ Tassos Georgiou ${ }^{1}$
}

To cite: Prokopiou E, Kolovos $P$, Georgiou C, et al. Omega-3 fatty acids supplementation protects the retina from age-associated degeneration in aged C57BL/6J mice. BMJ Open Ophthalmology 2019;4:e000326. doi:10.1136/ bmjophth-2019-000326

Received 2 May 2019 Revised 28 August 2019 Accepted 22 September 2019

Check for updates

(C) Author(s) (or their employer(s)) 2019. Re-use permitted under CC BY-NC. No commercial re-use. See rights and permissions. Published by BMJ.

${ }^{1}$ Ophthalmos Research and Educational Institute, Nicosia, Cyprus

${ }^{2}$ University of Nicosia Medical School, Nicosia, Cyprus

${ }^{3}$ Department of Electron Microscopy/Molecular Pathology, Cyprus Institute of Neurology and Genetics, Nicosia, Cyprus ${ }^{4}$ Bioinformatics Group, Cyprus Institute of Neurology and Genetics, Nicosia, Cyprus

${ }^{5}$ The Cyprus School of Molecular Medicine, Cyprus Institute of Neurology and Genetics, Nicosia, Cyprus

Correspondence to Dr Ekatherine Prokopiou; prokopiou.k@unic.ac.cy

Dr Tassos Georgiou; tassosgeorgiou@hotmail.com

\section{ABSTRACT}

Objective To evaluate the therapeutic effects of omega-3 $(\omega 3)$ fatty acids in the retina of aged mice when the blood arachidonic acid (AA)/eicosapentaenoic acid (EPA) ratio is maintained between 1.0 and 1.5. Methods and analysis Aged (24-month-old) wild-type C57BL/6J mice were allocated to two groups: $\omega 3$ treated and untreated. Treatment with $\omega 3$ was by daily gavage administration of EPA and docosahexaenoic acid for 60 days. Gas chromatography was used to identify and quantify fatty acids in the blood and retina. To count lipofuscin granules and measure the photoreceptor layer, eyecups were examined histologically using transmission electron microscopy and light microscopy. We also analysed eyecups using mass spectrometry-based proteomics.

Results AA levels were lower, and EPA levels were higher, in the blood and retinas of the $\omega 3$-treated group than in the untreated group, resulting in a lower AA/EPA ratio. The $\omega 3$-treated group also showed significantly fewer lipofuscin granules and a thicker outer nuclear layer than the untreated group. Proteomic analysis revealed significantly greater expression of myelin basic protein, myelin regulatory factorlike protein, myelin proteolipid protein and glial fibrillar acidic protein in the $\omega 3$-treated group than in the untreated group. Three different pathways were significantly affected by $\omega 3$ treatment: fatty acid elongation, biosynthesis of unsaturated fatty acids and metabolic pathways.

Conclusion Two months of $\omega 3$ supplementation (when the blood AA/EPA 1.0-1.5) in aged mice reduced lipofuscin granule formation in the retina and protected the photoreceptor layer, suggesting that $\omega 3$ supplementation slows normal age-related retinal degeneration.

\section{INTRODUCTION}

Visual function declines with age, as observed in the majority of apparently 'diseasefree' individuals who experience some degree of age-associated changes in vision. ${ }^{1}$ These changes include reductions in visual acuity, ${ }^{2}{ }^{3}$ colour perception, ${ }^{45}$ dark adaptation and contrast sensitivity. ${ }^{67}$ The prevention of age-related loss of vision or enhancement of visual acuity in elderly people would significantly improve their quality of life, in addition to improving their function in activities of daily living.

\section{Key messages}

What is already known about the subject?

> Visual decline in 'disease-free' individuals, who are over 65 years of age, can become apparent mainly through changes in visual acuity, dark adaptation and contrast sensitivity. These changes are inevitable in the majority of the elderly, unless some type of intervention can become available to delay them from occurring. Currently, there are no guidelines to suggest preventative measurements against age-associated retinal degeneration, apart from lifestyle modifications.

The protective effects of $\omega 3$ fatty acids against different eye pathologies have been demonstrated numerous times either in preclinical animal models or in human trials. Therefore, we hypothesised that $\omega 3$ fatty acids could be beneficial in preventing retinal degeneration and visual decline associated with ageing.

\section{What are the new findings?}

In the current study, we evaluated the effect of $\omega 3$ fatty acids (fish oil formulation of eicosapentaenoic acid (EPA):docosahexaenoic acid $(\mathrm{DHA})=5: 1)$ in healthy aged mice (24-month-old), while reaching and maintaining an arachidonic acid (AA)/EPA ratio within our target range (1.0-1.5). This ratio was emphasised and discussed in our previous reports which we believe it is an indication that the $\omega 3$ fatty acids are administered at the right dosage and that EPA is in high enough levels to get incorporated into the retina. This is of great importance because some previous attempts used $\omega 3$ fatty acids in patients with eye conditions including age-related macular degeneration and had inconclusive results.

$>$ Our data indicate that supplementation with $\omega 3$ fatty acids for 60 days results in a decreased number of lipofuscin granules within the retinal pigment epithelium and demonstrated a protective effect against photoreceptor age-related damage. Interestingly, an increase in myelin-related proteins was observed in the treatment group which may indicate that $\omega 3$ fatty acids have a regenerative potential through stimulation of myelogenesis.

Age-associated changes in visual acuity can be explained by two different theories: the 'biological clock theory' and the 'tear 


\section{Key messages}

\section{How might these results change the focus of research or} clinical practice?

- The findings of the current study suggest that $\omega 3$ supplementation can be considered a therapeutic intervention against ageassociated retinal dysfunction. The prevention of age-related loss of vision or enhancement of visual acuity in the elderly can significantly improve their quality of life, in addition to improving their function in activities of daily living.

theory'. ${ }^{8}$ The biological clock theory focuses on the genetic programming encoded within DNA, whereas the tear theory emphasises the importance of the physical and emotional stress to which we subject our bodies. For instance, diet (eg, the consumption of fat, sugar, caffeine and alcohol) is a major contributor to age-associated eye dysfunction because it can decrease the ability to repair cellular damage. Dietary supplementation could have a significant beneficial effect on this type of age-associated damage in the eyes. This dysfunction is due to a reduction in the local antioxidant enzymes found in the retinal pigment epithelium (RPE) along with a decrease in the macular pigment density. ${ }^{9}$ Such damage is usually derived from the production of free radicals that creates metabolic waste products, including a mixture of substances known as lipofuscin. ${ }^{1011}$

Lipofuscin can disrupt vital chemical and biological processes that are essential for cellular function. The major component of lipofuscin is N-retinylideneN-retinylethanolamine (A2E), a vitamin A-derived pyridinium bisretinoid isomer that is a byproduct of the visual cycle and may cause RPE damage. ${ }^{1213}$ In turn, RPE damage can lead to retinal degeneration ${ }^{14}$ and eventually to vision loss.

Age-related functional and anatomical changes are more likely to reflect alterations in the retina, probably associated with the loss and attenuation of RPE cells ${ }^{15}$ and photoreceptors (mostly rods). ${ }^{16}$ Additional anatomical changes may be observed: the trabecular endothelium is lost from the trabecular meshwork, ${ }^{17}$ the cornea becomes flattened, ${ }^{18}$ the lens grows and becomes cataractous, ${ }^{19} 20$ Bruch's membrane thickens, ${ }^{21}$ the ciliary body becomes collagenised $^{19}$ and choroidal vascular changes occur. ${ }^{22}$ In addition, degenerative processes are observed, such as vitreous liquefaction ${ }^{23}$ and the accumulation of drusen. ${ }^{21}{ }^{24}$ The optic nerve exhibits structural changes with age, including the loss of nerve fibre bundles and the accumulation of proteoglycan. ${ }^{25}$

Age is a major risk factor for diseases of the retina such as age-related macular degeneration (AMD),${ }^{26}$ the most common cause of irreversible blindness in the elderly population in industrialised countries. ${ }^{27}$ Although ageing does not directly lead to the occurrence of AMD,${ }^{28}$ it plays a major role in the pathophysiology of this condition due to the accumulation of oxidised lipoproteins and free radicals in the retina and choroid. ${ }^{29} 30$
Retinas in young and aged individuals and in patients with AMD showed multiple changes in the morphology and distribution of astrocytes, ${ }^{31}$ which are glial cells that communicate with neurons and the surrounding connective tissue. In addition to being involved in numerous supportive processes, astrocytes have a high antioxidant content, ${ }^{32}$ as well as the potential to protect neurons from free radicals. ${ }^{33}$

Aged retinas demonstrated fewer astrocytes and spaces with no glial fibrillar acidic protein (GFAP) reactivity in the nerve fibre layer (due to the disappearance of the processes from the astrocytes located in this layer). Furthermore, astrocytes from aged retinas have more cytoplasmic organelles and glial filaments than astrocytes from younger retinas. ${ }^{31}$ The number of chambers in the astroglial plexus reduces increasingly with age, as demonstrated by a comparison of persons aged 60-79 and persons aged over $80 .{ }^{31}$ Large numbers of reactive astrocytes were reported in all patients with AMD, which revealed that these astrocytes phagocytosed the residue from ganglion cells that died via apoptosis/necrosis. More lipofuscin deposits were observed in the aged retinas than in the younger retinas; however, pathological retinas from patients with AMD had even more deposits of lipofuscin granules. ${ }^{31}$

Similarities in the physiology and cell biology of ageing in humans and rodents have been observed; for example, 2.5-year-old mice show decreases in the maximum voltage obtained from electroretinograms, while opsin gene transcription, cone density and increment thresholds are unchanged even in extremely old mice, ${ }^{34}$ just as in humans. The number of rods and the length of their outer segment decreases with age, as does the total level of retinal opsin. The scotopic visual acuity, contrast sensitivity and $\mathrm{a}$ and $\mathrm{b}$ waves from rod electroretinogram recordings are also reduced in aged mice. ${ }^{35}$

Samuel et al compared all major retinal neuronal types in young adult (3-5 months old) and aged (24-28 months old) $\mathrm{C} 57 \mathrm{BL} / 6 \mathrm{~J}$ mice. ${ }^{36}$ The study revealed that the total retinal area expanded with age, whereas the retinal ganglion cell dendritic arbours, and the size and complexity of the retinal ganglion cell axonal arbours, decreased with age. This finding suggests that the transmission of visual signals to the brain is compromised. In addition, age-related structural changes were observed in the amacrine and bipolar cells. ${ }^{36}$ Taking into account the morphological and molecular changes that occur in aged C57BL/6J mice, and the similarities they have with human aged eyes, we considered these mice an appropriate animal model for the purpose of the present study

The protective effects of omega-3 ( $\omega 3$ ) polyunsaturated fatty acids (PUFAs) against retinal damage have been demonstrated in several disease states, including pathologies associated with inflammation, ischaemia, light and oxygen. ${ }^{37}$ Furthermore, the treatment of retinal degeneration with $\omega 3$ PUFAs has shown promise in animal models, through the reduction of A2E accumulation and preservation of retinal functions. ${ }^{38} 39$ 
$\omega 3$ PUFAs are known to reduce inflammation, primarily through resolvins that are derived from eicosapentaenoic acid (EPA, C20:5 n-3) and docosahexaenoic acid (DHA, C22:6 n-3), and through anti-inflammatory mediators. ${ }^{40}$ DHA activates a number of nuclear hormone receptors that modulate proinflammatory genes, including the peroxisome proliferator-activated receptor alpha. ${ }^{37}$ EPA-rich diet reduces choroidal neovascularisation in C57BL/6J mice, through a suppression of intercellular adhesion molecule, monocyte chemotactic protein 1 , vascular endothelial growth factor and interleukin $6 .{ }^{41}$ Proinflammatory eicosanoids, including prostaglandins and leukotrienes that are involved in leucocyte chemotaxis and inflammatory cytokine production, are generated from the omega-6 ( $\omega 6)$ PUFA arachidonic acid (AA, C20:4 n-6). ${ }^{42}$ (13 PUFAs also have the capacity to affect production and activation of angiogenic growth factors, AA-based vasoregulatory eicosanoids and metalloproteinases. ${ }^{37}$ Finding the right balance between the proinflammatory and anti-inflammatory mediators produced by $\omega 3$ and $\omega 6$ PUFAs plays a key role in the resolution of inflammatory responses and therefore possibly in age-associated vision loss.

The therapeutic potential of $\omega 3$ PUFAs in eye pathologies has also been demonstrated by our research team in several animal models. The fish oil formulation that we use in our studies is rich in EPA. Previous reports demonstrated that EPA competes with AA and significantly inhibits AA oxygenation in vitro, thus reducing activity in the inflammatory pathways involving prostaglandins $\mathrm{D}, \mathrm{E}$ and $\mathrm{F}^{4344}$

The activity of $\omega 3$ was tested in a rat model of anterior ischaemic optic neuropathy. Our findings showed that the positive effect of $\omega 3$ on the retina is mediated by several different actions, including preventing retinal ganglion cell apoptosis, decreasing inflammatory cell infiltration and regulating macrophage polarisation, which decreases cytokine-induced injury of the optic nerve. ${ }^{45}$

Similarly, Prokopiou et al reported the effects of $\omega 3$ PUFAs in the CCL2 ${ }^{-/-}$mouse model of retinal degeneration. A protective response, perhaps with a regenerative potential, was observed in $\omega 3$-treated animals, with a pronounced increase in the size of the photoreceptor outer nuclear layer (ONL) and a reduction in interleukin 18. ${ }^{46}$ Kalogerou et al demonstrated the effectiveness of $\omega 3$ PUFAs in the DBA/2J glaucoma model; retinal ganglion cells were protected against cell death and a reduced inflammatory response was observed. ${ }^{47}$

Recently, the therapeutic potential of $\omega 3$ was also reported by Prokopiou et al in the $\mathrm{ABCA}^{-/-}$mouse model of Stargardt disease. ${ }^{48}$ In particular, significantly less A2E and fewer lipofuscin granules were observed in $\omega 3$-treated animals than in untreated animals, and the photoreceptor ONL was preserved. Proteomic analysis revealed lower levels of complement component 3 in the treatment group, indicative of a decreased complementinduced inflammatory response. Importantly, in all these studies, the levels of particular PUFAs (including EPA and AA) were examined during treatment, allowing the treatment dosage to be adjusted in order to achieve and maintain a blood AA/EPA level of $1.0-1.5$. We emphasise the importance of detecting these fatty acids (FAs) in our previous reports. ${ }^{46}{ }^{48}$ Lower levels of EPA and higher levels of AA, in either circulating blood or the retina, are associated with some retinopathies, including AMD. ${ }^{4-51}$ Monitoring such systemic biomarkers during experimental animal investigations or in human trials could provide a good indication of disease progression and treatment success.

We hypothesise that $\omega 3$ PUFAs would be beneficial for disease-free individuals who experience some degree of age-associated changes in vision. Therefore, in the present study, we investigated how the administration of 13 PUFAs (primarily EPA and some DHA), to 2-year-old C57BL/6J wild-type mice, affects RPE cells and photoreceptors.

\section{MATERIALS AND METHODS \\ Animals}

Young (3-month-old) and aged (24-month-old) C57BL/6 J wild-type mice (inbred at the animal facility at the Cyprus Institute of Neurology and Genetics) of equal amount of both sexes were used in this study; the mice were kept under standard housing conditions on a 12 hours/12 hours dark/light cycle with food and water provided ad libitum. Standard diet was provided (Mucedola, Milano, Italy). Mean body weight of animals prior to treatment was $28.5 \pm 2.5 \mathrm{~g}$. The general appearance of the mice was monitored daily, and body weight measurements were recorded weekly.

\section{Study design}

The animals were allocated to three different groups ( $\mathrm{n}=15$ /group): young untreated; aged untreated and aged treated with $\omega 3$ PUFAs. The aged mice were randomly allocated to the latter two groups. The animals in the treatment group received the supplement daily by gavage for 60 days. The total oral dosing volumes of the gavage administration did not exceed $10 \mathrm{~mL} / \mathrm{kg}(\sim 0.3$ $\mathrm{mL} /$ mouse). The $\omega 3$ treatment was a fish oil formulation that contained $571 \mathrm{mg} / \mathrm{mL}$ EPA and $114 \mathrm{mg} / \mathrm{mL}$ DHA (Eyetas, TAS Ophthalmos L.C., Nicosia, Cyprus); therefore, each treatment administration had $172 \mathrm{mg}$ EPA and $34 \mathrm{mg}$ DHA per $0.3 \mathrm{~mL}$. The supplement dosage was adjusted in order to achieve the blood AA/EPA within the range of 1.0-1.5. At the end of the study, the mice were euthanised by cervical dislocation.

\section{FA analysis}

To determine the AA/EPA ratio before, during and on completion of treatment, we collected blood samples $(\mathrm{n}=5$ mice) from the tail of each mouse in each group on day zero (D0), day 30 (D30) and day 60 (D60). Blood samples from young mice were only collected on D60 for comparison purposes. Additionally, on study 
completion (D60), both retinas from three animals per group were collected and analysed for the same purpose. Samples were collected on Whatman (GE Healthcare, Life Sciences, Buckinghamshire, UK) filter paper and stored at $-20^{\circ} \mathrm{C}$ until ready for analysis. Samples were processed as previously described ${ }^{485}$; briefly, they were separated into organic and aqueous phases, where the organic phase was collected, redissolved in n-hexane and analysed using a gas chromatography flame ionisation detector.

An Agilent GC-6890 system (Hewlett Packard Agilent Technologies, California, USA) was equipped with a flame ionisation detector. The conditions, temperatures of the injection port and detector were set as previously described. ${ }^{48} 52$ The injection volume was $1 \mathrm{~mL}$ in the splitless injection mode. A capillary column (DB-23 fusedsilica capillary, $30 \mathrm{~m} \times 0.25 \mathrm{~mm}$ internal diameter, $0.25 \mu \mathrm{m}$ film thickness; Supelco, Bellefonte, Pennsylvania, USA) was used.

\section{Transmission electron microscopy (TEM)}

Electron microscopy was used to quantify lipofuscin granules in the RPE cells. For this analysis, the cornea and lens from five animals per group were removed, and the dissected eyecups were fixed, dehydrated and embedded in an epon and araldite resin mixture (Agar Scientific, Essex, UK), as previously described. ${ }^{48}$ Ultrathin $(80 \mathrm{~nm})$ sections were prepared using a Reichert Jung ultramicrotome (Leica, Wetzlar, Germany). Sections with a silver-gold interference colour were mounted on 200mesh copper grids (Agar Scientific) and contrasted with uranyl acetate and lead citrate. Images were obtained on a JEM 1010 TEM (JEOL, Tokyo, Japan) equipped with a Mega View III digital camera (Olympus, Hamburg, Germany).

As the appearance of lipofuscin is somewhat variable, we used a previous morphological definition to identify lipofuscin granules. ${ }^{4853}$ Melanosomes that were fused with lipofuscin were considered melanolipofuscin granules and were included in the combined category of 'lipofuscin and melanolipofuscin'. We used ImageJ software (http://imagej.nih.gov/ij, National Institutes of Health, Bethesda, Maryland, USA) for lipofuscin and melanolipofuscin quantification. For each image, we determined the total area of RPE cytoplasm as previously reported. ${ }^{48}$ The extent of the lipofuscin/melanolipofuscin area is expressed as the number of square micrometres occupied by lipofuscin per $1000 \mu^{2}$ of RPE cytoplasm.

\section{Light microscopy}

To assess how the treatment affected retinal photoreceptors (ie, ONL thickness), we examined the same eyes that were used for the quantification of lipofuscin granules, under a Leica DMi8 light microscope (Leica). ONL measurements were made at different locations around the entire retinal section (centre, middle and periphery). Image J was used to calculate the ONL thickness at each location, and the mean ONL thickness around the entire retina was compared among the different groups.

\section{Mass spectrometry-based proteomics}

To further assess the effect of treatment, proteomic analysis was conducted on eyecup extracts. The cornea and lens from five eyes per group (one eye/mouse) were removed, and the dissected eyecups were immediately snap frozen and stored at $-80^{\circ} \mathrm{C}$ until needed. The frozen eyecups were analysed as previously described. ${ }^{48}$ Briefly, the samples were lysed, and once centrifuged, the supernatant was collected and the proteins were precipitated in acetone overnight and subsequently resuspended in urea buffer. The protein concentration was determined using the bicinchoninic acid protein assay, and the samples were further processed using a modified filteraided sample preparation protocol. ${ }^{54}$ Peptides were analysed on a Waters Synapt G2Si high-definition mass spectrometer (Waters) operated in ion-mobility mode using an ultradefinition mass spectrometry approach..$^{55}$ Each sample was analysed in triplicate. The raw mass spectrometry data were analysed using Progenesis QI for proteomics V.3.0 (Waters) and proteins identified using the UniProt mouse reference proteome database (http://www.uniprot.org/, January 2018 version, containing 16889 reviewed proteins). The PANTHER Classification System V.12.0 (http://pantherdb.org/) was used to determine the functional classification of the identified proteins. Proteins showing an increase $(>1.5$ fold) or a decrease $(<0.66$-fold) were further analysed using DAVID Bioinformatics Resources V.6.8 (https:// david.ncifcrf.gov/).

\section{Statistical analysis}

All statistical analyses were performed using GraphPad Prism statistical software (GraphPad Software, USA, V.5). Data are expressed as the means \pm SEMs. A twotailed Student's t-test or one-way analysis of variance with Tukey's post hoc test was used to evaluate the differences between groups. Statistical significance was set at $\mathrm{p}<0.05$.

\section{Patient and public involvement}

Patients were not involved in this study.

\section{RESULTS}

\section{General observations}

No abnormalities were noted in the general appearance of the mice, and no significant changes in body weight were found over time in any of the groups. No sex differences were observed at any stage of the analysis.

\section{(13 PUFAs increase blood EPA and decrease blood AA}

Blood was collected from mice in different groups $(n=5)$ on D0 (prior to the initiation of treatment), on D30, and at the end of the study on D60, to determine the percentage of total $\omega 3$ PUFAs (EPA and DHA) and $\omega 6$ PUFAs (AA and dihomo- $\gamma$-linolenic acid, DGLA; figure 1). Blood samples from young mice were only collected on D60, since no differences were observed at these ages (1, 2 and 

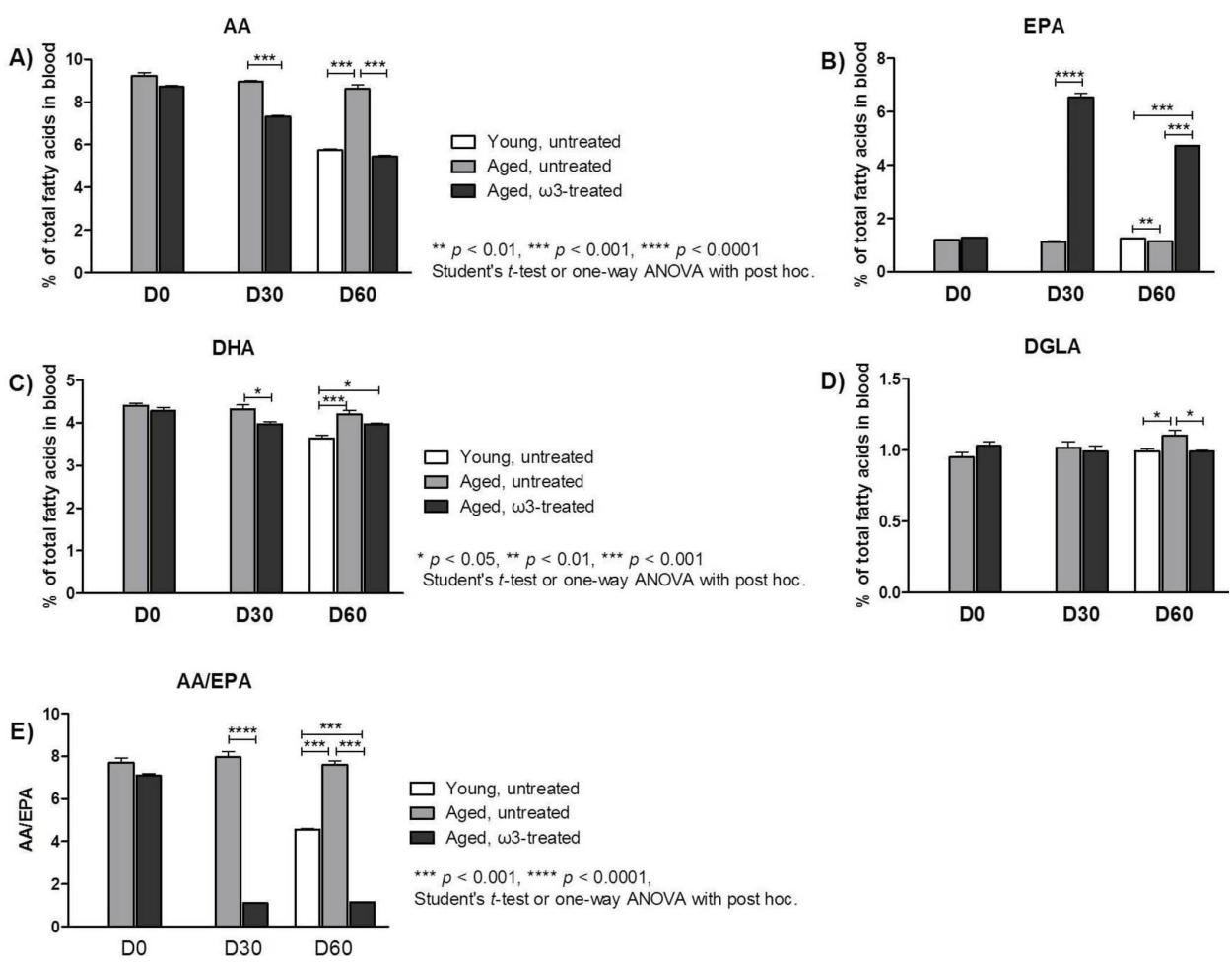

Figure 1 (A)-(E) Fatty acid analysis. Percentages of total (A) arachidonic acid (AA), (B) eicosapentaenoic acid (EPA), (C) docosahexaenoic acid (DHA) and (D) dihomo- $\gamma$-linolenic acid (DGLA) in blood samples collected on D0, D30 and D60. (E) The AA/EPA ratio in each group at D0, D30 and D60. The data represent the means $\pm S E M s(n=5$ mice per group). ANOVA, analysis of variance.

3 months old) in previous pilot studies we performed. The most important finding was that EPA level as a percentage of total blood FAs was about six times higher in the $\omega 3$-treated group $(6.55 \pm 0.15, \mathrm{p}<0.0001)$ than in the aged untreated group $(1.13 \pm 0.15)$ on D30, a difference that was maintained to D60. The percentage of AA was lower on D30 in the aged treated group $(7.30 \pm 0.07$, $\mathrm{p}<0.001)$ than in the aged untreated group $(8.96 \pm 0.05)$, and this difference was even greater on D60 $(5.44 \pm 0.05$, $\mathrm{p}<0.001$ vs $8.61 \pm 0.20)$. Therefore, on D30, the blood $\mathrm{AA} / \mathrm{EPA}$ ratio was more than seven times lower in the $\omega 3$-treated animals $(1.1 \pm 0.02, \mathrm{p}<0.0001)$ than in the aged untreated (7.98 \pm 0.26$)$ animals. At the end of the study on $\mathrm{D} 60$, the $\mathrm{AA} / \mathrm{EPA}$ ratio in the $\omega 3$-treated mice remained unchanged from that on D30 and was still lower than the $\mathrm{AA} / \mathrm{EPA}$ ratio in the young untreated mice $(4.56 \pm 0.05$, $\mathrm{p}<0.001)$. In the $\omega 3$-treated mice, a small reduction was observed in the percentage of DHA and DGLA on D30 and D60. Therefore, $\omega 3$-treated mice demonstrated lower AA and higher EPA levels in the blood, favourable of the desired AA/EPA ratio (1.0-1.5).

$\omega 3$ PUFAs increase retinal EPA levels and decrease retinal AA levels

Retinas from each group $(n=3)$ were collected at the end of the study on D60, and the constituent FAs (EPA, DHA, DGLA and AA) were quantified as a percentage of total FA content using gas chromatography (figure 2). We observed similar trends in the retinas as in the blood. Specifically, the percentage of retinal EPA in the aged $\omega 3$-treated group $(1.75 \pm 0.04, \mathrm{p}<0.01)$ was significantly higher than that in the aged untreated group $(1.23 \pm 0.00)$. Retinal EPA was undetectable in the young untreated animals. Conversely, the AA level in the treatment group $(5.58 \pm 0.01, \mathrm{p}<0.05)$ was significantly lower than that in the aged untreated group $(6.56 \pm 0.28)$, whereas the AA
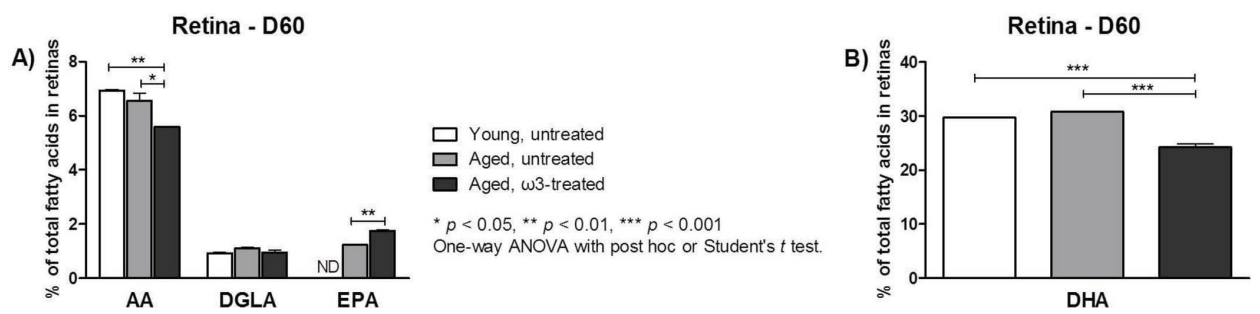

Figure 2 Fatty acid analysis of retina samples collected on D60. The percentage of (A) arachidonic acid (AA), dihomo- $\gamma$ linolenic acid (DGLA), eicosapentaenoic acid (EPA) (ND: not detected) and (B) docosahexaenoic acid (DHA) is expressed as the mean \pm SEM ( $n=3$ per group). ANOVA, analysis of variance. 
A)

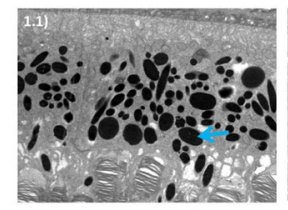

Young, untreated

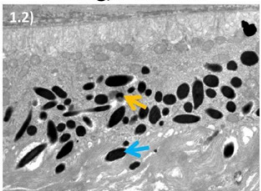

Aged, untreated
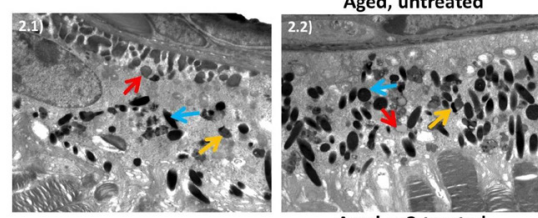

Aged, w3-treated
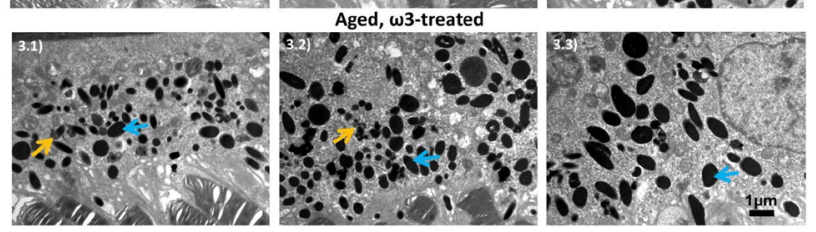

B)

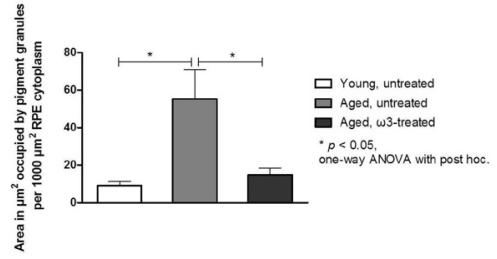

Figure 3 (A) Representative transmission electron microscopy micrographs of retinal pigment epithelium (RPE) cells from the three groups. Blue arrows: melanosomes; red arrows: irregularly shaped, electron-dense material (most likely lipofuscin granules); yellow arrows: granules of irregular shape appeared to fuse with melanosomes (melanolipofuscin granules). The electron density of these confluent granules was occasionally as high as that of melanosomes (original magnification 10000x, scale bar: $1 \mu \mathrm{m}$ ). (B) Quantification of lipofuscin and melanolipofuscin granules by electron microscopy. The total area $\left(\mu \mathrm{m}^{2}\right)$ occupied by lipofuscin and melanolipofuscin per $1000 \mu^{2}$ section of RPE cytoplasm was significantly lower in aged mice treated with $\omega 3$ than in aged untreated mice. The data represent the means \pm SEMs ( $n=5$ per group). ANOVA, analysis of variance.

level in the young untreated animals $(6.93 \pm 0.04)$ was similar to that in the aged untreated animals, but significantly higher than that in the treated animals $(\mathrm{p}<0.01)$. DHA was the most abundant FA in the retina, with a level much higher than that of any other FA, as previously reported. ${ }^{56}$ A significantly lower level of DHA was observed in the aged $\omega 3$-treated group $(24.25 \pm 0.64)$ compared with the aged untreated $(30.86 \pm 0.02, \mathrm{p}<0.001)$ and young untreated $(29.70 \pm 0.15, \quad \mathrm{p}<0.001)$ groups (figure 2B).

\section{$\omega 3$ PUFAs reduce the number of lipofuscin granules}

The RPE layer was examined using electron microscopy to assess the ultrastructural differences between the young untreated, aged untreated and aged $\omega 3$-treated animals (figure 3). Melanosomes (figure 3A, blue arrows) were abundant in all groups and were uniformly electron dense, not fused with other organelles, and spindleshaped, ovoid or round. An accumulation of irregularly shaped, electron-dense material in the cytoplasm of RPE

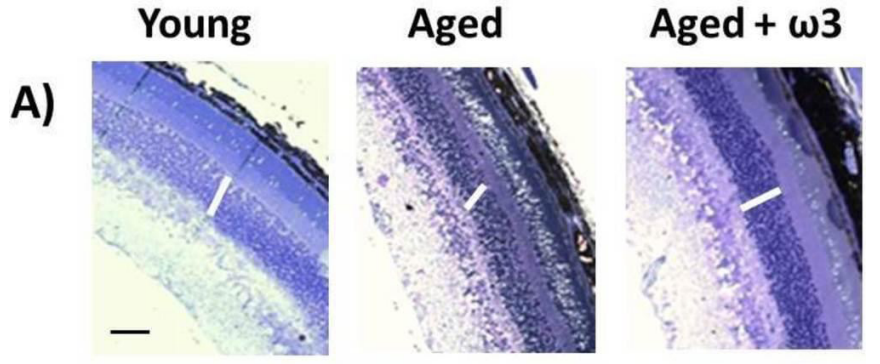

B)

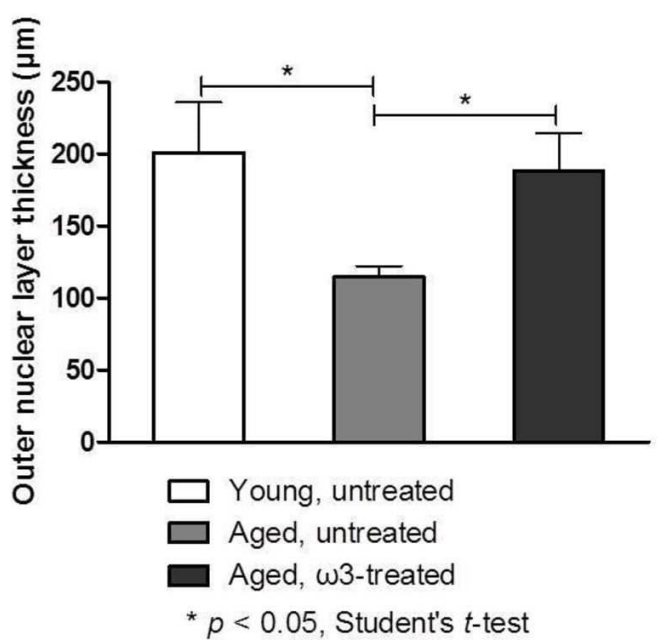

Figure 4 (A) Representative optical photomicrographs from the three groups. Samples were examined with a light microscope (original magnification 200x, scale bar $=150$ $\mu \mathrm{m})$. The white lines denote the retinal outer nuclear layer (ONL). (B) Measurements of ONL thickness were performed at three different locations around the entire retinal section (including peripheral and central retina). The data represent the means \pm SEMs ( $n=5$ eyes per group).

cells was mostly noted in the aged untreated animals (figure 3A, red arrows). This type of organelle might be similar to typical lipofuscin granules. Melanolipofuscin granules (figure 3A, yellow arrows) were more frequently observed and were therefore included in the quantification analysis (figure $3 \mathrm{~B})$. The total area $\left(\mu \mathrm{m}^{2}\right)$ occupied by lipofuscin and melanolipofuscin per 1000 $\mu^{2}$ section of RPE cytoplasm was six-fold higher in the aged untreated $\left(55.27 \pm 15.58 \mu^{2}, \mathrm{p}<0.05\right)$ than in the young untreated $\left(9.03 \pm 2.11 \mu^{2}\right)$ mice. This total area in the $\omega 3$-treated group $\left(14.76 \pm 3.65 \mu^{2}, \mathrm{p}<0.05\right)$ was significantly (approximately four-fold) smaller than that in the aged group and, interestingly, was comparable to that in the young group.

\section{(13 PUFAs and the photoreceptor layer}

The ONL thickness represents the long-term health of photoreceptor nuclei; therefore, we captured images (figure 4A) and quantified the ONL thickness in the three groups (figure 4B). In the young untreated mice, the ONL thickness was $201.0 \pm 35.0 \mu \mathrm{m}$, whereas in the aged untreated mice, it was significantly thinner: $114.5 \pm 7.7 \mu \mathrm{m}(\mathrm{p}<0.05)$. The ONL in $\omega 3$-treated aged 


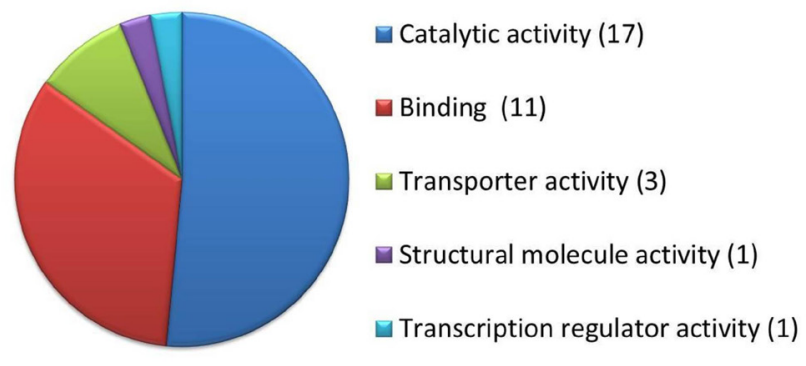

Figure 5 Functional classification of significantly affected proteins following treatment with $\omega 3$ fatty acids, as determined by PANTHER classification system V.12.0. The number of proteins involved in each category is indicated in brackets.

mice was significantly greater in thickness $(188.0 \pm 26.0$ $\mu \mathrm{m}, \mathrm{p}<0.05)$ than in the aged untreated mice, and did not significantly differ in thickness to the ONL of young untreated mice.

\section{(13 PUFAs and proteomic analysis}

Proteomic analysis enabled us to identify and quantify 2786 proteins in the eyecups. The levels of approximately 49 of these proteins differed significantly $(p<0.05)$ among the three groups. According to PANTHER functional classification, the total number of function hits was 33, as shown in figure 5 , with the majority of these proteins exhibiting catalytic activity (including isomerase, ligase, acetyltransferase and decarboxylase activity). A smaller proportion of the proteins that differed significantly between groups has binding activity or is involved in processes such as transport (ie, membrane organisation, cation and lipid transportation).
Notably, among the proteins that differed significantly within the groups, there were some that may play a key role in the progression of age-associated degeneration. In particular, myelin basic protein (MBP), myelin proteolipid protein (MPP) and myelin regulatory factor-like protein (MRFLP) were analysed separately. Figure 6 shows the fold change defined as the level of each protein detected in each group divided by the measured level of that protein in the young untreated group. Overall, significantly greater fold changes in MBP, MPP and MRLFP levels were observed in the aged $\omega 3$-treated group $(2.17 \pm 0.57,1.89 \pm 0.32$ and $1.49 \pm 0.22$, respectively; $\mathrm{p}<0.05)$ than in the aged untreated group $(0.57 \pm 0.06$, $0.71 \pm 0.04$ and $0.47 \pm 0.06$, respectively). The fold changes in the levels of MBP and MPP in the $\omega 3$-treated group were also significantly higher than those in the young untreated group $(\mathrm{p}<0.05)$.

In addition to differences between the groups in myelin-related proteins, we also determined the fold change of GFAP, an astrocyte marker, in the three groups (figure 7). There was a two-fold reduction in the level of GFAP in the aged untreated mice $(0.55 \pm 0.05$, $\mathrm{p}<0.05)$ compared with that in the young untreated mice $(1.00 \pm 0.11)$. Interestingly, there was a twofold increase in the $\omega 3$-treated group $(1.18 \pm 0.10, \mathrm{p}<0.01)$ compared with the aged untreated group. The fold change in GFAP level in the $\omega 3$-treated group was comparable to that in the young untreated group.

To identify specific pathways that were significantly altered following treatment with $\omega 3$, further analysis of the proteins that were expressed differently between the groups was performed. Specifically, three pathways demonstrated a difference between the treatment and control groups: pathways involved in FA elongation (acyl-CoA thioesterase 1, Acot2, Acot3, Acot5, $\mathrm{p}=9.5 \mathrm{E}^{-06}$ ), biosynthesis of unsaturated FAs (Acot1, Acot2, Acot3,
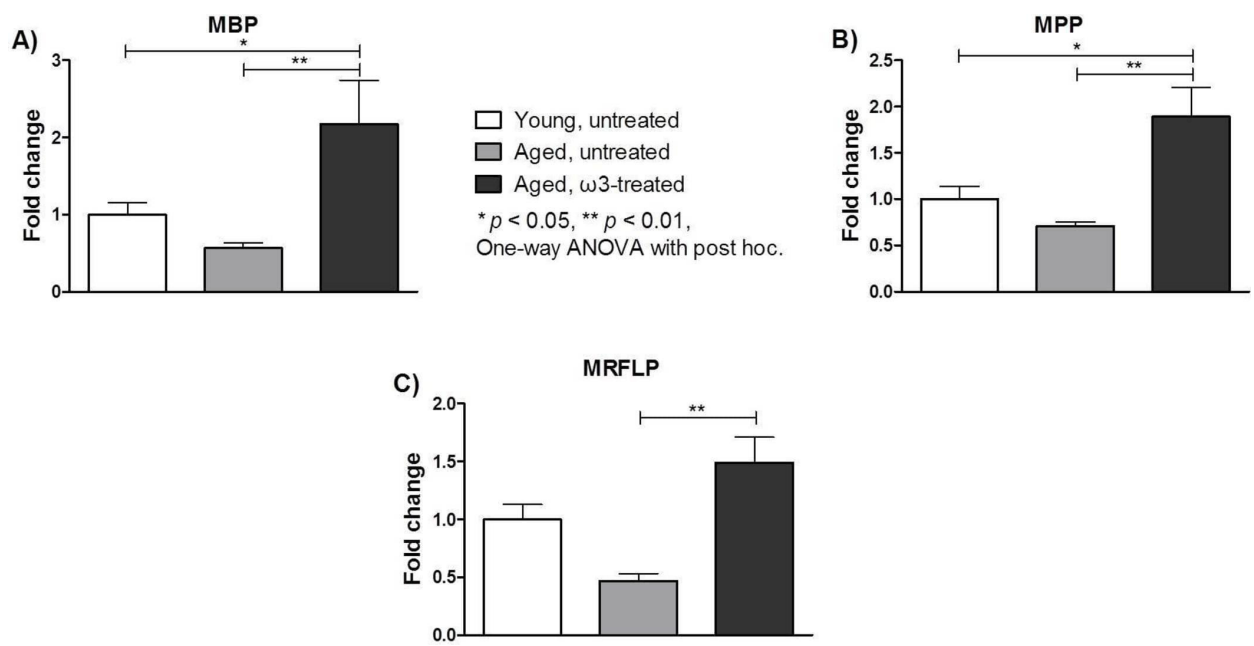

Figure 6 Fold changes of (A) myelin basic protein (MBP), (B) myelin proteolipid protein (MPP) and (C) myelin regulatory factor-like protein (MRFLP) in two eyecups per animal from five animals per group. The data are expressed as the fold change compared with the measured level of each protein in the young untreated group and represent the means \pm SEMs $(n=5$ per group). ANOVA, analysis of variance. 


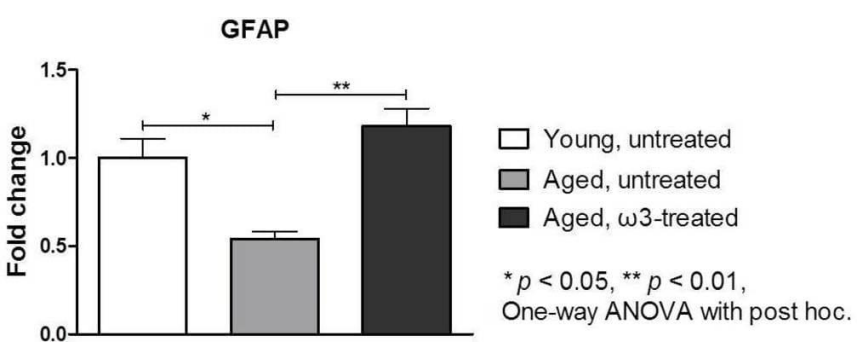

Figure 7 Fold changes of glial fibrillar acidic protein (GFAP) in two eyecups per animal, from five animals per group. The data are expressed as the fold change compared to the measured level of GFAP in the young untreated group and represent the means \pm SEMs ( $n=5$ per group). ANOVA, analysis of variance.

Acot5, $\mathrm{p}=1.07 \mathrm{E}^{-05}$ ) and metabolism (subunit of the oligosaccharyltransferase complex, homolog A, glutamate decarboxylase-like $1, \mathrm{p}=0.049$ ).

\section{DISCUSSION}

Age-associated changes in vision, primarily a reduction in visual acuity, are unavoidable in the majority of 'diseasefree' individuals. To date, no treatment has been robustly shown to prevent, slow or reverse vision loss in the aged population. Therefore, new therapeutics or supplements are needed for improving the quality of life of affected individuals.

In recent studies, particular emphasis has been placed on the role of $\omega 3$ PUFAs in eye diseases. The positive effect of $\omega 3$ treatment was investigated in animal models of retinal and optic nerve pathologies ${ }^{454452}$ and in clinical studies of AMD and dry eyes. ${ }^{57-59}$ Although the results were not conclusive, we investigated the effect of $\omega 3$ PUFAs in age-associated retinal degeneration in healthy mice. We hypothesised that the most beneficial effect would be achieved when using a dosage that maintains an AA/EPA ratio between 1.0 and 1.5 and an appropriate formulation of $\omega 3$ PUFAs (EPA:DHA=5:1).

Our findings show that treatment with 13 PUFAs, when $\mathrm{AA} / \mathrm{EPA}$ is maintained between 1.0 and 1.5, reduced the accumulation of lipofuscin granules and increased the level of myelin-related proteins and the number of astrocytes (as indicated by an increase in GFAP). Demonstrating a protective effect against photoreceptor damage would require a considerably longer experiment than our resources permitted; even so, compared with no treatment in aged mice, 2 months of treatment with $\omega 3$ PUFAs in aged mice resulted in a notable preservation of the photoreceptor layer. If this effect can be confirmed in the long term, supplementation with $\omega 3$ PUFAs could be considered a preventive measure for age-associated vision loss. In particular, EPA appears to have a critical role in protecting the retina, since its percentage was significantly increased in the retina, whereas the percentage of DHA was not.

Serhan et al have extensively studied the mechanism of action of $\omega 3$ PUFAs. ${ }^{60-62}$ The processes involved are complex, and primarily implicate the generation of anti-inflammatory mediators-for example, resolvins, which are generated from the $\omega 3$ PUFAs EPA and DHA via cyclooxygenase/lipoxygenase pathways. Resolvins promote the control of inflammation in neural tissues and reduce the levels of proinflammatory eicosanoids generated from AA. ${ }^{60-62}$ Although the level of resolvins in blood and retina would have been beneficial for the purpose of this study, several attempts were made to detect them without any success, perhaps due to their short half-life.

During the $\omega 3$ supplementation treatment and at completion of the study, we observed that the AA/EPA ratio was maintained at an average of 1.0-1.5, which was within our target range. The reduction in the ratio was due to the increase in EPA (from the fish oil formulation), and due to the reduction in AA observed in both the blood and the retinal tissue. Our present and previous studies also show that EPA successfully incorporates into the retinal tissue. ${ }^{48} 52$ DHA is known to be expressed abundantly in photoreceptors ${ }^{56}$; this was confirmed by the high levels of DHA compared with the levels of the other FAs in the retina.

Additional mechanisms unrelated to inflammation might be involved in the mechanism of action of $\omega 3$ PUFAs. Our retinal proteomic analysis showed that three different pathways were significantly affected by treatment: FA elongation, biosynthesis of unsaturated FAs and metabolic pathways. These alterations were not surprising, since supplementation with dietary $\omega 3$ PUFAs is known to increase the elongation of particular FAs. ${ }^{63} 64$

In addition, the present data suggest that treatment with $\omega 3$ PUFAs may confer regenerative potential through the stimulation of myelin-related proteins, such as MBP, MRFLP and MPP. Our findings (increased MBP, MRFLP and MPP protein levels) agree with previous reports of myelogenesis being affected by environmental interferences including nutrition, with $\omega 3$-rich diets inducing an increased myelin synthesis. ${ }^{65}$ Further evidence demonstrated that EPA stimulates the expression of myelin proteins in the rat brain. ${ }^{66}$ Specifically, a single dose of EPA or DHA increased the MBP, MPP and myelin oligodendrocyte protein mRNA levels in nearly all brain regions in 2-day-old rats; this effect was more pronounced in EPA-treated rats. ${ }^{66}$ In contrast, $\mathrm{AA}$ was shown to decrease the phosphorylation of MBP in cultured rat oligodendrocytes via either a diffusible paracrine signal or a signal transduction mechanism, thus reducing MBP's activity. ${ }^{67}$ Furthermore, Salvati et al reported that EPA but not AA stimulates the expression of proteolipid protein via cAMP-mediated pathways in a time-dependent and concentration-dependent manner in C6 glioma cells. ${ }^{68}$ Our results confirm that $\omega 3$ PUFAs stimulate proteins essential for myelination in the retina, and suggest that they might furthermore prevent retinal degeneration.

Our proteomic analysis revealed that GFAP protein expression was elevated after $\omega 3$ treatment, indicating that the treatment increased the number of astrocytes. As 
confirmed in our animal model, astrocytes show a degenerative profile during normal ageing, implying a reduction in antioxidant and protective benefits to the host. Thus, by increasing astrocyte abundance via $\omega 3$ PUFAs administration, neuroprotection of the eye may be established. A similar effect was recently demonstrated by treating anterior horn cells in the spinal cord of male albino rats with $\omega 3$ PUFAs plus aspartame; rats treated with aspartame alone showed a higher rate of cell death and fewer GFAP-positive astrocytes. ${ }^{69}$ In addition, Zgórzyńska et al showed that the incubation of rat primary astrocytes with DHA and EPA improves the antioxidant defense in astrocytes via an Nrf2-dependent mechanism, determined by the ratio of DHA to EPA incorporated into membrane phospholipids. ${ }^{70}$ Together, these findings suggest that supplementation with $\omega 3$ PUFAs increases the number of astrocytes in the retina and may protect neurons against age-associated retinal degeneration.

Interest in how dietary $\omega 3$ PUFAs influence several physiological (eg, ageing) or pathological (eg, cardiovascular disease) conditions has grown considerably over the past decade. Numerous clinical studies have focused mainly on the anti-inflammatory properties of $\omega 3$ PUFAs as well as on their neuroprotective and regenerative effects. ${ }^{71-74}$ In particular, one review analysed human brain MRI studies and experimental studies and confirmed that $\omega 3$ PUFAs can protect the brain during ageing by preventing peroxidation, inflammation and excitotoxicity; modulating neurotransmission; and increasing neurogenesis, thereby improving the brain's 'coping' mechanisms during ageing. ${ }^{75}$

We have established here that $\omega 3$ PUFAs have protective and therapeutic potential against age-related decline in visual function in 2-year-old-aged C57BL/6J mice. This was demonstrated by treated mice showing a lower number of lipofuscin granules, preservation of the retinal structure (ie, the photoreceptor layer), greater expression of myelin-related proteins and a higher level of GFAP expression (indicating an increased number of astrocytes). Although future work would be valuable, our results suggest that $\omega 3$ PUFAs may be considered a protective dietary supplement against the vision loss caused by normal ageing.

Contributors EP and PK designed and performed the experiments. MK, LP, CG and KS contributed to some of the experimental procedures. TG supplied the reagents/materials/analysis tools. TG and KK provided guidance and support. EP analysed the data and wrote the manuscript. All contributing authors have read and approved the final version of the manuscript.

Funding This research was supported by Ophthalmos Research and Educational Institute (Nicosia, Cyprus) and was not supported by any specific grant from funding agencies in the public, commercial, or not-for-profit sectors.

Competing interests TG has a patent on the use of omega-3 fatty acids in eye diseases.

Patient consent for publication Not required.

Ethics approval The animal care and experimental procedures were performed in accordance with the EU Directive 2010/63/EU for animal experiments and approved by the Cyprus Government's Chief Veterinary Officer.

Provenance and peer review Not commissioned; externally peer reviewed.
Open access This is an open access article distributed in accordance with the Creative Commons Attribution Non Commercial (CC BY-NC 4.0) license, which permits others to distribute, remix, adapt, build upon this work non-commercially, and license their derivative works on different terms, provided the original work is properly cited, appropriate credit is given, any changes made indicated, and the use is non-commercial. See: http://creativecommons.org/licenses/by-nc/4.0/.

ORCID iD

Ekatherine Prokopiou http://orcid.org/0000-0002-8466-2065

\section{REFERENCES}

1 Spear PD. Neural bases of visual deficits during aging. Vision Res 1993;33:2589-609.

2 Weale RA. Senile changes in visual acuity. Trans Ophthalmol Soc U K 1975;95:36-8.

3 Del Viva MM, Agostini R. Visual spatial integration in the elderly. Invest Ophthalmol Vis Sci 2007;48:2940-6.

4 Ohta $\mathrm{Y}$, Kato $\mathrm{H}$. Color perception changes with age: test results by p-N anomaloscope. Mod Probl Ophthalmol 1976;17:345-2.

5 Page JW, Crognale MA. Differential aging of chromatic and achromatic visual pathways: behavior and electrophysiology. Vision Res 2005;45:1481-9.

6 Domey RG, McFARLAND RA, Chadwick E. Dark adaptation as a function of age and time. II. A derivation. J Gerontol 1960;15:267-79.

7 Werner JS. Night vision in the elderly: consequences for seeing through a "blue filtering" intraocular lens. $\mathrm{Br} \mathrm{J}$ Ophthalmol 2005;89:1518-21.

8 Salvi SM, Akhtar S, Currie Z. Ageing changes in the eye. Postgrad Med J 2006;82:581-7.

9 Beatty S, Koh H, Phil M, et al. The role of oxidative stress in the pathogenesis of age-related macular degeneration. Surv Ophthalmol 2000;45:115-34.

10 Dorey CK, Wu G, Ebenstein D, et al. Cell loss in the aging retina. Relationship to lipofuscin accumulation and macular degeneration. Invest Ophthalmol Vis Sci 1989;30:1691-9.

11 Bindewald A, Bird AC, Dandekar SS, et al. Classification of fundus autofluorescence patterns in early age-related macular disease. Invest Ophthalmol Vis Sci 2005;46:3309-14.

12 Sparrow JR, Zhou J, Ben-Shabat S, et al. Involvement of oxidative mechanisms in blue-light-induced damage to A2E-laden RPE. Invest Ophthalmol Vis Sci 2002;43:1222-7.

13 Anderson OA, Finkelstein A, Shima DT. A2E induces IL-1B production in retinal pigment epithelial cells via the NLRP3 inflammasome. PLoS One 2013;8:e67263.

14 Fishkin NE, Sparrow JR, Allikmets R, et al. Isolation and characterization of a retinal pigment epithelial cell fluorophore: an all-trans-retinal dimer conjugate. Proc Natl Acad Sci U S A 2005;102:7091-6.

15 Streeten BW. Development of the human retinal pigment epithelium and the posterior segment. Arch Ophthalmol 1969;81:383-94.

16 Jackson GR, Owsley C, Curcio CA. Photoreceptor degeneration and dysfunction in aging and age-related maculopathy. Ageing Res Rev 2002;1:381-96

17 McMenamin PG, Lee WR, Aitken DA. Age-Related changes in the human outflow apparatus. Ophthalmology 1986;93:194-209.

18 Edelhauser HF. The balance between corneal transparency and edema the Proctor lecture. Invest. Ophthalmol. Vis. Sci. 2006;47:1755-67.

19 Folberg R. The eye, in Ophthalmic Pathology. In: Spencer W, ed. An atlas and textbook. Philadelphia: W. B. Saunders, Co, 1996: 1-37.

20 Eagle WS. The lens, in Ophthalmic Pathology. In: Spencer W, ed. An atlas and textbook. Philadelphia: W. B. Saunders, Co, 1996: 72-437.

21 Green W. Retina, in Ophthalmic Pathology. In: Spencer W, ed. An Atlas and Textbook. Philadelphia: W. B. Saunders, Co, 1996: 667-1331.

22 Ramrattan RS, van der Schaft TL, Mooy CM, et al. Morphometric analysis of Bruch's membrane, the choriocapillaris, and the choroid in aging. Invest Ophthalmol Vis Sci 1994;35:2857-64.

23 Spencer W. Vitreous, in Ophthalmic Pathology. In: Spencer W, ed. An atlas and textbook. Philadelphia: W. B. Saunders, Co, 1996: 623-66.

24 Spraul CW, Grossniklaus HE. Characteristics of drusen and Bruch's membrane in postmortem eyes with age-related macular degeneration. Arch Ophthalmol 1997;115:267-73.

25. Rao WS NA. Optic nerve, in Ophthalmic Pathology. In: An atlas and textbook. Philadelphia: W. B. Saunders, Co, 1996: 513-622.

26 Jackson GR, Owsley C. Visual dysfunction, neurodegenerative diseases, and aging. Neurol Clin 2003;21:709-28. 
27 Klein R, Peto T, Bird A, et al. The epidemiology of age-related macular degeneration. Am J Ophthalmol 2004;137:486-95.

28 Young RW. Pathophysiology of age-related macular degeneration. Surv Ophthalmol 1987:31:291-306.

29 Nag TC, Wadhwa S. Ultrastructure of the human retina in aging and various pathological states. Micron 2012;43:759-81.

30 Harman D. Aging: a theory based on free radical and radiation chemistry. J Gerontol 1956;11:298-300.

31 Ramírez JM, Ramírez Al, Salazar JJ, et al. Changes of astrocytes in retinal ageing and age-related macular degeneration. Exp Eye Res 2001;73:601-15.

32 Wilson JX. Antioxidant defense of the brain: a role for astrocytes. Can J Physiol Pharmacol 1997;75:1149-63.

33 Vernadakis $A$. Changes in astrocytes with aging. In: Federoff $S$, Vernadakys A, eds. Biochemistry, and pharmacology of astrocytes. Orlando, FL, U.S.A: Academic Press, 1986: 377-407.

34 Williams GA, Jacobs GH. Cone-based vision in the aging mouse. Vision Res 2007;47:2037-46.

35 Kolesnikov AV, Fan J, Crouch RK, et al. Age-Related deterioration of rod vision in mice. J Neurosci 2010;30:11222-31.

36 Samuel MA, Zhang Y, Meister M, et al. Age-Related alterations in neurons of the mouse retina. J Neurosci 2011;31:16033-44.

37 SanGiovanni JP, Chew EY. The role of omega-3 long-chain polyunsaturated fatty acids in health and disease of the retina. Prog Retin Eye Res 2005;24:87-138.

38 Dornstauder B, Suh M, Kuny S, et al. Dietary docosahexaenoic acid supplementation prevents age-related functional losses and $\mathrm{A} 2 \mathrm{E}$ accumulation in the retina. Invest Ophthalmol Vis Sci 2012:53:2256-65.

39 Ramkumar HL, Tuo J, Shen DF, et al. Nutrient supplementation with N3 polyunsaturated fatty acids, lutein, and zeaxanthin decrease A2E accumulation and VEGF expression in the retinas of Ccl2/Cx3cr1-deficient mice on Crb1rd8 background. J Nutr 2013;143:1129-35.

40 Calder PC. Omega-3 fatty acids and inflammatory processes. Nutrients 2010;2:355-74.

41 Koto T, Nagai N, Mochimaru H, et al. Eicosapentaenoic acid is antiinflammatory in preventing choroidal neovascularization in mice. Invest Ophthalmol Vis Sci 2007;48:4328-34.

42 Serhan CN, Gotlinger K, Hong S, et al. Resolvins, docosatrienes, and neuroprotectins, novel omega-3-derived mediators, and their aspirin-triggered endogenous epimers: an overview of their protective roles in catabasis. Prostaglandins Other Lipid Mediat 2004;73:155-72.

43 McCappin SC, Vandongen R, Croft KD. The effect of dietary eicosapentaenoic acid on arachidonic acid incorporation and metabolism in rat leukocytes. Prostaglandins 1987;34:505-17.

44 Wada M, DeLong CJ, Hong YH, et al. Enzymes and receptors of prostaglandin pathways with arachidonic acid-derived versus eicosapentaenoic acid-derived substrates and products. J Biol Chem 2007;282:22254-66.

45 Georgiou T, Wen Y-T, Chang C-H, et al. Neuroprotective effects of omega-3 polyunsaturated fatty acids in a rat model of anterior ischemic optic neuropathy. Invest Ophthalmol Vis Sci 2017;58:1603-11.

46 Prokopiou E, Kolovos P, Kalogerou M, et al. Therapeutic potential of omega- 3 fatty acids supplementation in a mouse model of dry macular degeneration. BMJ Open Ophthalmol 2017; 1 .

47 Kalogerou M, Kolovos P, Prokopiou E, et al. Omega-3 fatty acids protect retinal neurons in the DBA/2J hereditary glaucoma mouse model. Exp Eye Res 2018;167:128-39.

48 Prokopiou E, Kolovos P, Kalogerou M, et al. Omega-3 fatty acids supplementation: therapeutic potential in a mouse model of Stargardt disease. Invest Ophthalmol Vis Sci 2018;59:2757-67.

49 Gorusupudi A, Liu A, Hageman GS, et al. Associations of human retinal very long-chain polyunsaturated fatty acids with dietary lipid biomarkers. J Lipid Res 2016:57:499-508.

50 Sawada T, Tsubata H, Hashimoto N, et al. Effects of 6-month eicosapentaenoic acid treatment on postprandial hyperglycemia, hyperlipidemia, insulin secretion ability, and concomitant endothelia dysfunction among newly-diagnosed impaired glucose metabolism patients with coronary artery disease. an open label, single blinded, prospective randomized controlled trial. Cardiovasc Diabetol 2016;15:121.

51 Okada K, Kotani K, Yagyu H, et al. Eicosapentaenoic acid/ arachidonic acid ratio and smoking status in elderly patients with type 2 diabetes mellitus. Diabetol Metab Syndr 2014;6.
52 Prokopiou E, Kolovos P, Kalogerou M, et al. Therapeutic potential of omega-3 fatty acids supplementation in a mouse model of dry macular degeneration. BMJ Open Ophthalmol 2017;1:e000056.

53 Charbel Issa P, Barnard AR, Singh MS, et al. Fundus autofluorescence in the Abca4(-/-) mouse model of Stargardt disease--correlation with accumulation of A2E, retinal function, and histology. Invest Ophthalmol Vis Sci 2013;54:5602-12.

54 Wiśniewski JR, Zougman A, Nagaraj N, et al. Universal sample preparation method for proteome analysis. Nat Methods 2009;6:359-62.

55 Distler U, Kuharev J, Navarro P, et al. Drift time-specific collision energies enable deep-coverage data-independent acquisition proteomics. Nat Methods 2014;11): :167-70.

56 Birch EE, Birch DG, Hoffman DR, et al. Dietary essential fatty acid supply and visual acuity development. Invest Ophthalmol Vis Sci 1992;33:3242-53.

57 Chew EY, Clemons T, SanGiovanni JP, et al. The age-related eye disease study 2 (AREDS2): study design and baseline characteristics (AREDS2 report number 1). Ophthalmology 2012;119:2282-9.

58 Epitropoulos AT, Donnenfeld ED, Shah ZA, et al. Effect of oral reesterified omega-3 nutritional supplementation on dry eyes. Cornea 2016;35:1185-91.

59 Korobelnik J-F, Rougier M-B, Delyfer M-N, et al. Effect of dietary supplementation with lutein, zeaxanthin, and $\omega-3$ on macular pigment: a randomized clinical trial. JAMA Ophthalmol 2017:135:1259-66.

60 Serhan CN. Novel lipid mediators and resolution mechanisms in acute inflammation: to resolve or not? Am J Pathol 2010;177:1576-91.

61 Ariel A, Serhan CN. Resolvins and protectins in the termination program of acute inflammation. Trends Immunol 2007;28:176-83.

62 Serhan CN, Chiang N, Van Dyke TE. Resolving inflammation: dual anti-inflammatory and pro-resolution lipid mediators. Nat Rev Immunol 2008;8:349-61.

63 Tu WC, Cook-Johnson RJ, James MJ, et al. Omega-3 long chain fatty acid synthesis is regulated more by substrate levels than gene expression. Prostaglandins Leukot Essent Fatty Acids 2010;83:61-8.

64 Brenna JT, Salem N, Sinclair AJ, et al. Alpha-Linolenic acid supplementation and conversion to n-3 long-chain polyunsaturated fatty acids in humans. Prostaglandins Leukot Essent Fatty Acids 2009;80:85-91.

65 Di Biase A, Salvati S. Exogenous lipids in myelination and myelination. Kaohsiung J Med Sci 1997;13:19-29.

66 Salvati S, Natali F, Attorri L, et al. Eicosapentaenoic acid stimulates the expression of myelin proteins in rat brain. J Neurosci Res 2008;86:776-84

67 Takeda M, Soliven B. Arachidonic acid inhibits myelin basic protein phosphorylation in cultured oligodendrocytes. Glia 1997;21:277-84.

68 Salvati S, Natali F, Attorri L, et al. Stimulation of myelin proteolipid protein gene expression by eicosapentaenoic acid in C6 glioma cells. Neurochem Int 2004;44:331-8.

69 Ali EMT, Sonpol HMA. Neuroprotective and ameliorating impacts of omega-3 against Aspartame-induced neuronal and astrocytic degeneration. Anat Rec 2017;300:1290-8.

70 Zgórzyńska E, Dziedzic B, Gorzkiewicz A, et al. Omega-3 polyunsaturated fatty acids improve the antioxidative defense in rat astrocytes via an Nrf2-dependent mechanism. Pharmacol Rep 2017:69:935-42

71 Kiecolt-Glaser JK, Belury MA, Andridge R, et al. Omega-3 supplementation lowers inflammation in healthy middle-aged and older adults: a randomized controlled trial. Brain Behav Immun 2012;26:988-95

72 Davinelli S, Corbi G, Righetti S, et al. Cardioprotection by cocoa polyphenols and $\omega-3$ fatty acids: a Disease-Prevention perspective on aging-associated cardiovascular risk. J Med Food 2018;21:1060-9.

73 Clark CM, Monahan KD, Drew RC. Omega-3 polyunsaturated fatty acid supplementation reduces blood pressure but not renal vasoconstrictor response to orthostatic stress in healthy older adults. Physiol Rep 2018;6:e13674.

74 Hooper C, De Souto Barreto P, Pahor M, et al. The relationship of omega 3 polyunsaturated fatty acids in red blood cell membranes with cognitive function and brain structure: a review focussed on Alzheimer's disease. J Prev Alzheimers Dis 2018;5:78-84.

75 Denis I, Potier B, Heberden C, et al. Omega-3 polyunsaturated fatty acids and brain aging. Curr Opin Clin Nutr Metab Care 2015:18:139-46. 